\title{
Surveiller les animaux et contrôler les citoyens, ou comment policer les bêtes pour mieux hiérarchiser les humains entre 1789 et $1799 . .$.
}

Controlling Animals and Monitoring Citizens, or How to Police Beasts to Better

Organize Humans between 1789 and 1799

\section{Pierre Serna}

Traducteur : Stephen Clay et Hannah Callaway

\section{(2) OpenEdition} Journals

Édition électronique

URL : https://journals.openedition.org/ahrf/13280

DOI : $10.4000 /$ ahrf. 13280

ISSN : 1952-403X

Éditeur :

Armand Colin, Société des études robespierristes

Édition imprimée

Date de publication : 15 octobre 2014

Pagination : 109-144

ISBN : 978-2-20-092927-5

ISSN : 0003-4436

Référence électronique

Pierre Serna, «Surveiller les animaux et contrôler les citoyens, ou comment policer les bêtes pour mieux hiérarchiser les humains entre 1789 et 1799... », Annales historiques de la Révolution française [En ligne], 377 | juillet-septembre 2014, mis en ligne le 22 octobre 2014, consulté le 01 juillet 2021. URL http://journals.openedition.org/ahrf/13280 ; DOI : https://doi.org/10.4000/ahrf.13280 


\title{
SURVEILLER LES ANIMAUX ET CONTRÔLER LES CITOYENS, OU COMMENT POLICER LES BETTES POUR MIEUX HIÉRARCHISER LES HUMAINS ENTRE 1789 ET 1799...
}

Pierre SERNA

\begin{abstract}
L'article propose une étude inédite de la police des animaux dans Paris à partir des procès-verbaux des commissaires des sections déposés aux Archives de la Préfecture de Paris. L'enquête révèle l'omniprésence des animaux dans la capitale et les soucis qu'ils causent aux représentants de l'ordre. Source d'accidents, au cœur des interrogations sur l'amélioration de la salubrité publique, objets de commerce lorsqu'ils sont transformés en viande de boucherie, les animaux représentent aussi des enjeux politiques. La façon dont les hommes les traitent devient un sujet d'inquiétude également car elle exprime un rapport à l'inférieur, que le spectacle urbain peut rapidement transformer en objet de tensions sociales. Ordre et santé publics, spectacles républicains, régulation du commerce, protection des plus faibles, constituent autant de questions que la police des animaux révèle, dans le quotidien du policier au service du Nouveau Régime.
\end{abstract}

Mots-clés : police, Paris, animaux, boucherie, cheval, spectacle urbain, santé publique, accident.

\section{La scène initiale}

Un lecteur du journal La Décade, le journal qui contribue durant le Directoire au programme de régénération républicaine, raconte un fait divers dont il a été témoin et acteur. Trois chevaux ne parvenant à tirer une charge au haut de la rue de la Harpe, et ce malgré les invectives de leur charretier, l'auteur avec d'autres citadins, pousse à la roue... au sommet de 
la côte, le limonier s'écroule... le charretier se déchaîne à coups de fouet et de bâton sur le cheval... malgré les critiques qui s'élèvent du groupe de personnes assemblées, rien n'y fait, la violence contre l'animal ne cesse pas. Lorsque l'arrivée d'une nouvelle actrice fait tout à coup changer de face la scène brutale.

«Une marchande de légumes que la curiosité avait arrêtée comme beaucoup d'autres, sort tout d'un coup de la foule, dépose sur une borne la hotte qu'elle portait sur son dos, jette sans proférer un seul mot, ses regards autour d'elle, se saisit d'un pavé qui lui tombe sous la main, fond comme un éclair sur le charretier, lui porte sous le nez le terrible caillou : « Frappe le donc à présent, ose le frapper, monstre inhumain !»; en même temps qu'elle lui arrache le fouet, le brise en quatre morceaux qu'elle fait voler à vingt pas d'elle. Étourdi par cette apostrophe inattendue, le charretier pâlit et recule devant le pavé qui serrait de près ses mâchoires. Tous les spectateurs battent des mains : un seul, bien poudré, bien frisé, s'avise de gourmander l'héroïne. «"Chacun devrait se mêler de ses affaires ; le cheval appartenait au charretier, il avait le droit d'en faire ce que bon lui semblait, de le tuer même si c'est son plaisir... ne parviendra-t-on jamais à inculquer ces principes à la populace ?" $»^{1}$.

Il faut tout de même préciser que le lecteur n'est autre que... le directeur adjoint de l'école vétérinaire, membre de l'Institut national, le citoyen Gilbert, figure de proue de la naissance d'une médecine républicaine à l'égard des animaux. Tout est résumé dans ce qui dépasse le cadre d'une simple anecdote. Le micro fait-divers devient une source d'où partent les fils d'une histoire économique, l'approvisionnement de Paris et la circulation des denrées vers les zones de marchés de la capitale, l'histoire sociale, la mixité du groupe assemblé montre de suite la tension politique qui fonde le socle du Directoire, construit à la suite des principes de 1789 sur la sacralité de la propriété. Ceux qui possèdent pourraient faire ce qu'ils veulent de leur bien, sans que la «populace »n'ait rien à dire. L'histoire de la violence est aussi en chantier dans cet extrait. Dans ce pays en guerre depuis six ans, dans ce Paris transformé en capitale de guerre, dans ce pays où les ravages de la pauvreté ne sont pas endigués et qui se souvient encore de ces émeutes meurtrières et des répressions antipopulaires de l'an III, où fixer le seuil de « supportabilité » de la violence, ici des coups portés sur les animaux, où l'historien doit-il placer le curseur de la faculté des 
passants dans la rue à accepter un spectacle des plus rudes et traumatisants, afin de comprendre leur sensibilité ? C'est surtout une très belle page d'histoire du genre qu'offre Gilbert. Face au silence gêné et un peu lâche du groupe d'hommes, une femme seule, avec courage s'interpose, défie le charretier et renverse la situation. Dans ce pays qui commence à être travaillé par la construction d'une nouvelle virilité issue des champs de bataille ou des actions mâles des sans-culottes parisiens, la leçon d'audace genrée s'impose et interroge l'historien, sur la place des femmes, comme possibles régulatrices des incidents de rue et de justice banale dans ce $\mathrm{cas}^{2}$. Enfin cette anecdote ouvre des perspectives de recherches nouvelles et largement à explorer encore sur l'histoire des animaux, mais bien plus que cela, dans le domaine d'une histoire des relations entre les hommes et les animaux au cour de la cité, et point seulement dans l'espace rural ${ }^{3}$. Bien plus, au lieu d'imaginer une anthropo-histoire des hommes avec les animaux qui reposerait sur le constat d'une stabilisation séculaire de ces relations à partir d'une fonctionnalité domestique imposée par l'homme à « sa propriété mobile, utile mais silencieuse »-c'est ainsi que je définis pour le moment l'animal de ville- il convient alors de poser la question des rapports révolutionnés des hommes et des animaux entre 1789 et 1799. Certes cette femme aurait pu agir ainsi en 1787, voire en 1803. Pourtant l'histoire courte de la Révolution commence à transformer le rapport à l'animal, à l'autre, à l'Autre, par la naissance d'une nouvelle sensibilité, par la naissance de la conscience d'un code civil pensé bien avant 1804, par la prise de conscience qu'une société ne se construit pas par la distinction du haut, mais aussi par indistinction égalitaire du bas.

Ainsi, à leur corps défendant, les trois chevaux puis le cheval battu deviennent les acteurs centraux, figures possibles de l'extrême précarité dans la cité. Sous les sans-culottes, sous les sans-paroles, ils sont les sans-rien-du-tout... et comme Gilbert, de par la position stratégique qu'il occupe dans la république des savants du Directoire, le note à la suite de l'article : la façon dont on traite les animaux dit la manière dont on considère les inférieurs... la rage du bourgeois devant la résistance du petit peuple parisien à protéger une figure du faible au nom d'un droit à la protection, d'un devoir de bienfaisance au corps souffrant de l'animal

(2) Ce qui bien évidemment n'implique pas que les femmes soient cantonnées dans ce rôle et qu'elles ne puissent à leur tour être vectrices de violence.

(3) Michel MORICEAU, entre autres, Histoire du méchant loup, 3000 attaques sur l'homme en France $X V^{e}-X X^{e}$ siècle, Paris, Fayard, 2007 ou bien L'élevage sous l'Ancien régime : les fondements agraires de la France moderne XVI $-X V I I I^{e}$ siècles, Paris, Sedes, coll. « Regards sur l'histoire », 1999. 
travailleur, exprimé dans l'acte souverain de cette femme citoyenne sans citoyenneté, résume la tension politique et sociale de ce qui pourrait n'être qu'une historiette.

Où est et que fait la police durant cette scène de désordre ? Elle est manifestement absente... pas comme toujours car les commissaires et agents connaissent bien l'ensemble des situations où humains et animaux se trouvent mêlés à un désordre urbain, qui peut parfois dégénérer et exprimer des tensions sociales, voire des oppositions politiques, dont l'animal constitue le prétexte. Les archives sont riches de cette volonté de la part de la police d'ordonner la ville en imposant le nouvel ordre aussi aux animaux.

Cet article se propose donc d'explorer dans les dix années 17891799, la place de la police des bêtes, ses évolutions, son amélioration et ses interventions originales, en contexte politique nouveau, révolutionnaire, puis républicain. La révolution peut être désordre dans sa phase initiale. La révolution est également l'invention d'un ordre civil, sociétal, municipal, urbain, économico-industriel nouveau ${ }^{4}$. Comment utiliser le paradigme animal pour étudier le nouvel ordre politique qui se met en place?

\section{Les données de l'enquête : compter, mesurer, peser.}

La recherche s'est construite à partir du dépôt bien connu des papiers de police des sections parisiennes consultables aux archives de la préfecture de police ${ }^{5}$. Le fonds peut être appréhendé par le catalogue Labat, cet archiviste qui construisit seul un registre de plus 55000 entrées donnant simplement le titre de l'enquête, la date et son numéro de classement, en trois colonnes, à partir des papiers des sections. 33897 entrées concernent la période allant du $1^{\text {er }}$ janvier 1790 au 19 brumaire an VIII. Afin de construire l'échantillon de l'enquête ici présentée, la lecture de tous les titres d'affaires a été nécessaire afin de dégager de façon systématique les procès-verbaux concernant les animaux, quels que soient leur mention, leur statut, leur état. Cette remarque méthodologique permet de comprendre immédiatement une évidence pour le policier, que l'historien suit : les

(4) Cf Bernard GAINOT et Vincent DENIS (dir.), Un siècle d'ordre public en Révolution (de 1789 à la Troisième République), Paris, Société des études robespierristes, Collection études révolutionnaires $\mathrm{n}^{\circ} 11,2009$.

(5) Léon LABAT, Répertoire des procès-verbaux de la Révolution française, Archives de la Préfecture de Police de Paris. Peu utilisé de façon systématique, rédigé avant 1871, il offre à condition d'être comparé avec ce que contiennent les cartons, afin de corriger les oublis de l'archiviste, une entrée précieuse sur la vie policière parisienne. 
animaux se répartissent en vivants et morts, puisqu'il a été fait le choix délibéré de considérer la boucherie, comme expression aussi d'un rapport à l'animal, et qui accapare une bonne partie du temps des policiers occupés à régler le marché des animaux morts.

633 enquêtes se sont dégagées que l'on peut présenter dans un premier temps de cette façon ${ }^{6}$. La proportion des animaux est faible, à peine $1,9 \%$. L'animal n'est pas donc pas le principal fauteur de troubles. Un procès-verbal relatif aux animaux est dressé tous les cinq jours en moyenne, contre neuf par jour pour l'ensemble de l'activité policière, occupée surtout à réprimer la petite délinquance et à inventer le nouvel ordre public. Pour autant ce faible pourcentage n'enlève rien à l'omniprésence des animaux. Sûrement une très longue proximité des hommes et des animaux en ville explique le savoir-faire des humains face aux animaux difficilement contrôlables. Dans la macro-histoire de la police, ils sont un élément de micro-histoire qui donne à voir tout le dispositif de prévention, de surveillance et de punition dans la cité.

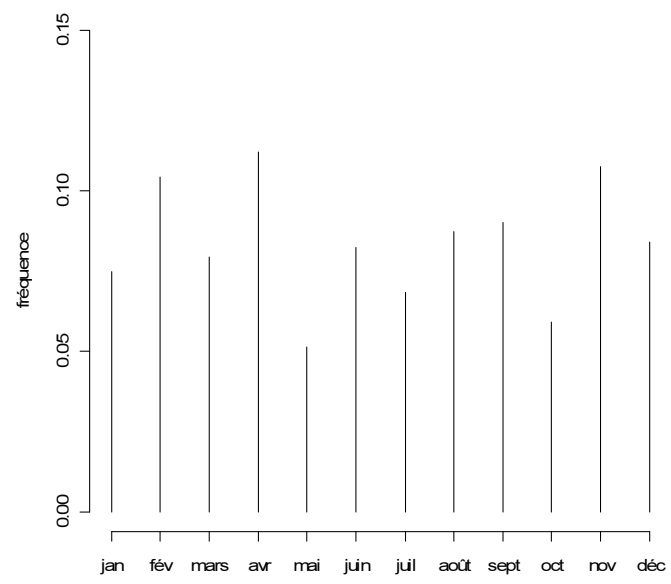

Figure 1. : Distribution mensuelle des procès-verbaux relatifs aux animaux

(6) Toute ma gratitude va vers Julien Alerini, Prag et docteur de l'Université de Paris I qui a bien voulu, à partir des données que je lui ai fournies, réaliser les schémas suivants. 
Dans l'année, le rythme de l'activité policière n'est pas constant. Trois moments émergent : février à avril (en particulier pour l'année 1794), août et septembre et enfin novembre et décembre, qui correspondent entre autres, à des moments de fortes entrées de bovins dans la cité, ou de contrôle particulier des bouchers en été 7 .

Tableau 1. : Animaux concernés par les procès-verbaux selon le mois.

\begin{tabular}{|l|l|l|l|l|l|l|l|l|l|l|l|l|}
\hline & janv & fév & mars & avr & mai & juin & juill & août & sept & oct & nov & déc \\
\hline Âne & 1 & 0 & 0 & 0 & 1 & 1 & 0 & 1 & 0 & 0 & 0 & 1 \\
\hline Boucherie & 9 & 27 & 19 & 21 & 4 & 9 & 11 & 8 & 13 & 6 & 5 & 5 \\
\hline Bovins & 4 & 2 & 8 & 16 & 0 & 2 & 2 & 2 & 6 & 2 & 27 & 7 \\
\hline Cheval & 24 & 26 & 19 & 25 & 16 & 31 & 22 & 27 & 23 & 22 & 28 & 34 \\
\hline Chien & 4 & 5 & 2 & 3 & 7 & 4 & 3 & 7 & 4 & 2 & 2 & 3 \\
\hline Cochon & 1 & 3 & 1 & 0 & 2 & 4 & 1 & 9 & 8 & 3 & 3 & 1 \\
\hline Exotique & 0 & 1 & 0 & 1 & 0 & 0 & 1 & 0 & 1 & 0 & 1 & 1 \\
\hline $\begin{array}{l}\text { Matière } \\
\text { Animale }\end{array}$ & 0 & 0 & 1 & 1 & 0 & 0 & 0 & 0 & 0 & 0 & 0 & 1 \\
\hline $\begin{array}{l}\text { Ovin- } \\
\text { Caprin }\end{array}$ & 0 & 1 & 0 & 0 & 2 & 0 & 1 & 1 & 0 & 1 & 1 & 0 \\
\hline $\begin{array}{l}\text { Pas } \\
\text { d'animal }\end{array}$ & 0 & 0 & 0 & 0 & 0 & 0 & 0 & 0 & 1 & 0 & 0 & 1 \\
\hline $\begin{array}{l}\text { Petits } \\
\text { animaux }\end{array}$ & 2 & 0 & 1 & 4 & 1 & 2 & 0 & 1 & 2 & 1 & 2 & 0 \\
\hline
\end{tabular}

Tableau 2. : Comparaison des fréquences des principaux animaux et de l'ensemble des PV animaliers.

\begin{tabular}{|l|l|l|l|l|l|l|l|l|l|l|l|l|}
\hline & janv & fév & mars & avr & mai & juin & juill & août & sept & oct & nov & déc \\
\hline Bovins & 6.6 & 19.7 & 13.9 & 15.3 & 2.9 & 6.6 & 8.0 & 5.8 & 9.5 & 4.4 & 3.6 & 3.6 \\
\hline Boucherie & 5.1 & 2.6 & 10.3 & 20.5 & 0.0 & 2.6 & 2.6 & 2.6 & 7.7 & 2.6 & 34.6 & 9.0 \\
\hline Chevaux & 8.1 & 8.8 & 6.4 & 8.4 & 5.4 & 10.4 & 7.4 & 9.1 & 7.7 & 7.4 & 9.4 & 11.4 \\
\hline $\begin{array}{l}\text { Ensemble } \\
\text { animaux }\end{array}$ & 7.1 & 10.3 & 8.1 & 11.2 & 5.2 & 8.4 & 6.5 & 8.8 & 9.2 & 5.8 & 10.9 & 8.5 \\
\hline
\end{tabular}

Quant aux chevaux, leur profil de distribution se distingue du profil global en juin et en décembre, cependant les écarts mensuels pour les chevaux sont insignifiants : omniprésent dans la ville, le cheval apparaît de manière équivalente d'un mois à l'autre.

(7) Rappelons que la fréquence mensuelle moyenne est de $8,3 \%$. 

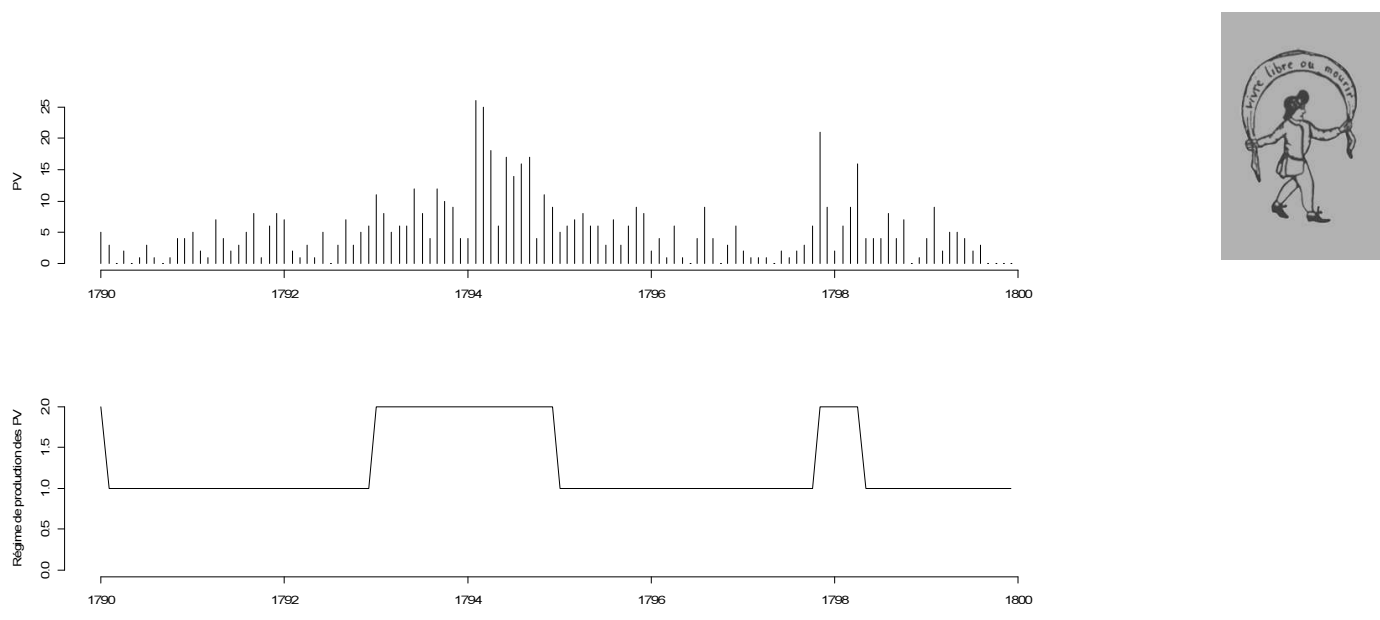

Figure 2. : Fréquence des procès-verbaux et régimes d'activités policière sur les animaux dans Paris (Sources APP : Catalogue Labat). ${ }^{\mathrm{a}}$

De fortes périodes d'activités alternent avec des moments de calme relatif, quatre moments se distinguent plus particulièrement, cœur de l'interrogation. Les deux premières périodes décrivent une activité moindre entre janvier 1790 et décembre 1792, puis de janvier 1795 à octobre 1797. En revanche deux phases d'activité soutenue sont repérables entre janvier 1793 et décembre 1794, puis de novembre à décembre 1797 et plongent sans tarder le chercheur au cœur du problème. En effet les deux phases à analyser révèlent deux moments de fortes tensions autour de la boucherie, des bovins dans la capitale et des problèmes qu'ils peuvent poser au policier. Dans le premier temps il s'agit durant la Convention et les années de crise de 1793-1794 du marché de la viande en temps de grave pénurie. Dans le second temps il s'agit d'un épisode d'épizootie, qui à notre connaissance est passé inaperçu dans les histoires de Paris, mais que seule la lecture croisée puis harmonisée des papiers des commissariats montre pour la première fois de façon précise et nouvelle ${ }^{8}$.

La deuxième donnée basique concerne l'espèce des animaux incriminés dans ces affaires de police 9 .

(8) Seule la publication des papiers du bureau du canton de police publiés par Alphonse Aulard, en donne la trace sans que les historiens de Paris n'aient décrit l'épisode.

(9) Voir en annexe la liste des dénominations animales. 


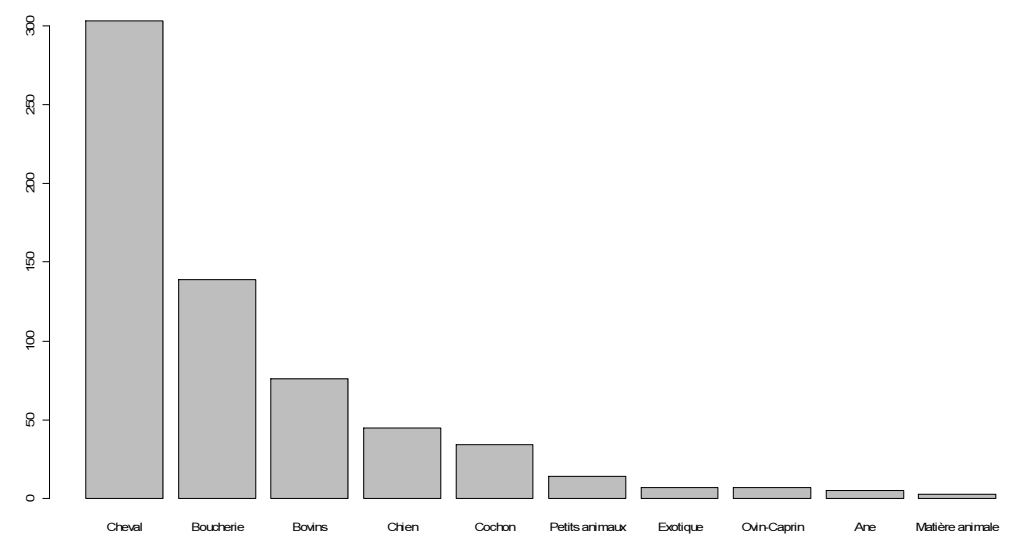

Figure 3. : Distribution des types d'animaux (source : APP/catalogue Labat).

La liste des animaux est longue et le catalogue dit le mélange dans Paris, capitale de France, de ces animaux de boucherie arrivant de Normandie et du Massif Central. Par ailleurs, la présence d'animaux domestiques exprime un nouveau rapport à l'animal et la banalité de cette présence aux côtés des citadins, mais aussi la place, fût-elle minoritaire, d'animaux exotiques confirme la circulation des marchandises et les effets de mode africaines, ou asiatique, voire américaine. Cependant et malgré la diversité des animaux que peut rencontrer le policier allant des perroquets, des singes, des bêtes fauves aux animaux familiers comme la volaille et les coqs, cinq groupes d'animaux se détachent nettement. Le cochon est toujours redouté dans ses divagations, le chien dont les morsures sont une inquiétude constante pour les parents, les bêtes à cornes dont les réactions incontrôlables dans les rues de Paris peuvent provoquer de lourds dégâts, ou dont l'état de santé accapare les agents. Se détachent enfin deux derniers groupes qui occasionnent moult soucis aux policiers, d'abord la viande de boucherie, omniprésente dans le travail du policier, et enfin, le fauteur de trouble par excellence, l'animal délinquant sans le savoir, responsable mais le plus souvent point coupable, le cheval omniprésent tout au long de la période $^{10}$.

(10) . Daniel Roche, La Culture équestre de l'Occident, XVI ${ }^{e} X I X^{e}$ siècle, L'ombre du cheval : Tome 1, Le cheval moteur, Essai sur l'utilité équestre, Paris, Fayard, 2008 et Histoire de la culture équestre, XVI $-X I X^{e}$ siècle, L'ombre du cheval : Tome 2, La gloire et la puissance, Paris, Fayard, 2011. 
Le troisième élément quantifié de la présence animale dans Paris pour le policier est le lieu d'intervention.

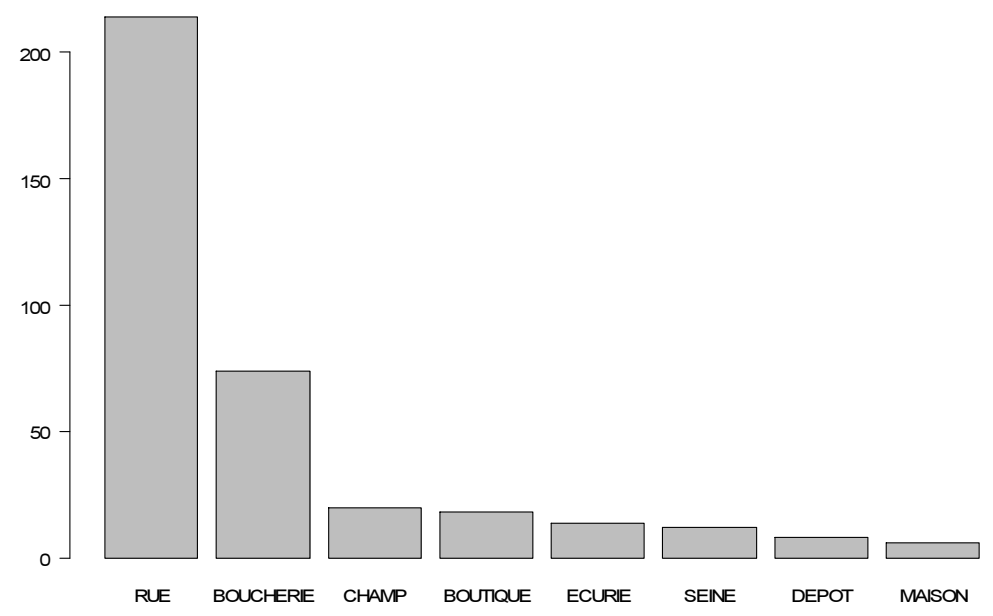

Figure 4. : Lieux de l'intervention policière. (source : APP/catalogue Labat).

Sans surprise, apparaît l'importance de la rue, lieu de l'action primordiale du policier garant de l'ordre public ${ }^{11}$. Le chercheur se trouve parfaitement dans le schéma décrit par Paolo Napoli et Vincent Millot lorsqu'ils décrivent le policier du XVIII ${ }^{\mathrm{e}}$ siècle qui fut un des agents les plus réformateurs de l'appareil d'État royal ${ }^{12}$. Cet agent se voit conférer une double fonction qui consiste d'abord à fluidifier autant que faire se peut toute forme de circulation en ville, ensuite à faciliter le plus possible le commerce sous toutes ses formes ainsi que des relations tranquilles de sociabilité . La responsabilité du policier peut se résumer à cette fonction : sécuriser la rue, pour que les circulations s'y déroulent le mieux possible. Il en va de la sécurité des habitants et de la prospérité des acteurs économiques. Dans

(11) Notons toutefois que dans $42,1 \%$ des procès-verbaux, le lieu de l'intervention n'est pas mentionné. Si la hiérarchie entre rue et boucherie est indéniable, les faibles effectifs et la petite distance entre les autres lieux empêchent d'établir une hiérarchie entre eux.

(12) Paolo NAPOLI, Naissance de la police moderne, Pouvoir, normes, sociétés, Paris, La Découverte, 2003 ;Vincent MiLlot (dir.) Les mémoires policiers 1750-1850. Études et pratiques policières du Siècle des Lumières au Second Empire, Rennes, PUR, 2006. 
ces conditions, le facteur animal est un souci majeur du policier, puisque les bêtes, difficilement raisonnables et a priori n'ayant pas intégré la peur du gendarme ou les impératifs des devoirs du vivre ensemble, ni royal ni républicain, incarnent des éléments potentiels de désordres urbains dont l'imprévisibilité provoque la contrariété du policier normatif. De façon logique par rapport aux tableaux précédents, la boucherie est l'autre lieu de contentieux entre les citadins et nécessite l'entrée du policier au plus près de l'étal mais aussi dans d'autres boutiques de mercandiers, ces revendeurs de viandes, forts surveillés. Ce schéma montre deux nouveaux espaces originaux : la Seine et les champs. Le cours d'eau, en plus de toutes ses fonctions propose un immense abreuvoir, ce qui n'est pas sans danger le plus souvent pour les jeunes garçons qui mènent les équidés nerveux pour s'abreuver. Paris est aussi une ville où les champs sont nombreux, où paissent des animaux, où de petits élevages, pourtant interdits, pullulent, où les chiens rôdent. Cela est vrai des champs mais il faut également penser à l'intérieur des maisons parfois également visitées. Comment ne pas ici penser à «l'homme aux lapins » décrit par Rétif de la Bretonne, et son élevage en galetas ${ }^{13}$ ?

La question de l'animal incontrôlable dans l'espace public, semipublic de la boutique et privé des maisons, pose plutôt, et le policier le sait bien, la question de l'animal incontrôlé. Depuis bien longtemps la bête n'est plus traînée en procès et son irresponsabilité en matière pénale renvoie directement au statut de son propriétaire ou de la personne qui, ne s'occupant pas correctement de l'animal, met en péril ses concitoyens. Dans 332 cas, le procès-verbal désigne un responsable d'accident ou de délit :

(13) Restif DE LA BRETONne, Les nuits de Paris, 1788-1789, réed., Paris, Bouquins, 1990, «L'homme aux lapins », p. 771. 


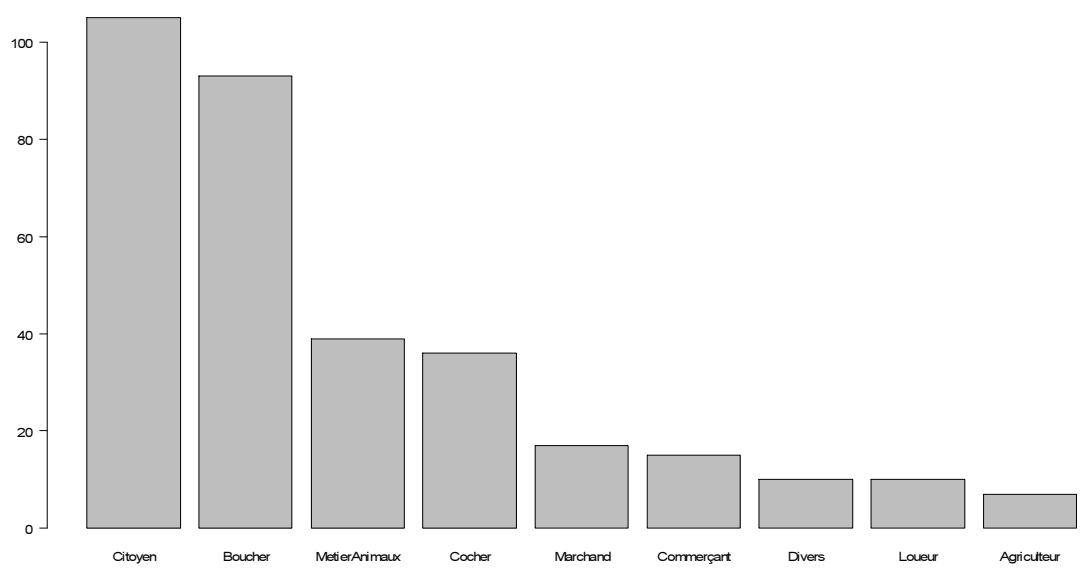

Figure 5. : Responsables des délits constatés et liés aux animaux. (source APP/Catalogue Labat $)^{\mathrm{a}}$

La catégorie « citoyen » est mal renseignée sur les procès-verbaux mais révèle le plus souvent les cavaliers imprudents, fauteurs d'accidents souvent graves contre les piétons. Le regroupement des métiers de cocher, de loueur, avec la catégorie des citoyens à cheval montre l'attention particulière des policiers face au principal moyen de transport de l'époque, qu'il soit individuel, qu'il serve à tracter une voiture pour une ou plusieurs personnes ou qu'il porte sur son bât plus qu'il n'en faut pour sa peine. La ville est chevaline au sens où l'économie du cheval, la présence du cheval et les dégâts causés par ceux qui en sont responsables, sont omniprésents dans le travail du policier. Ce tableau indique par là même les professions plus surveillées et donc davantage verbalisées, ce qui ne manque pas en retour de provoquer des tensions entre ces groupes fortement structurés que sont par exemple les cochers et les bouchers face aux policiers comme le révèlera la lecture précise des papiers. Qui dit responsable implique des victimes. 


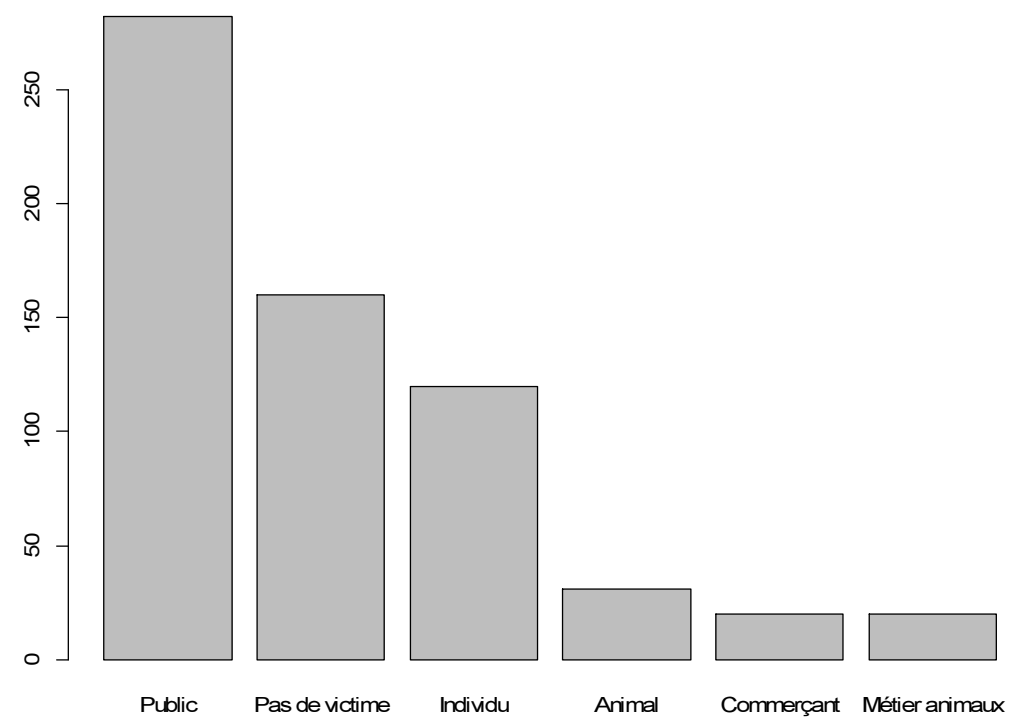

Figure 6. : Victimes désignées dans les procès-verbaux.

Le graphique est d'autant plus précieux qu'il indique clairement, contrairement à ce qu'une première lecture pourrait laisser croire que c'est le domaine public qui est la victime la plus souvent désignée. Les blessures parfois mortelles demeurent certes spectaculaires, mais le plus souvent c'est l'ordre urbain, les biens fonciers de la municipalité de Paris, l'ordre public qui sont abîmés ou bouleversés par les agissements des animaux mal dirigés par leurs maîtres ou leurs responsables.

Il resterait une dernière indication à donner, intéressante et décevante à la fois : mais où sévissent les animaux dans Paris ? 


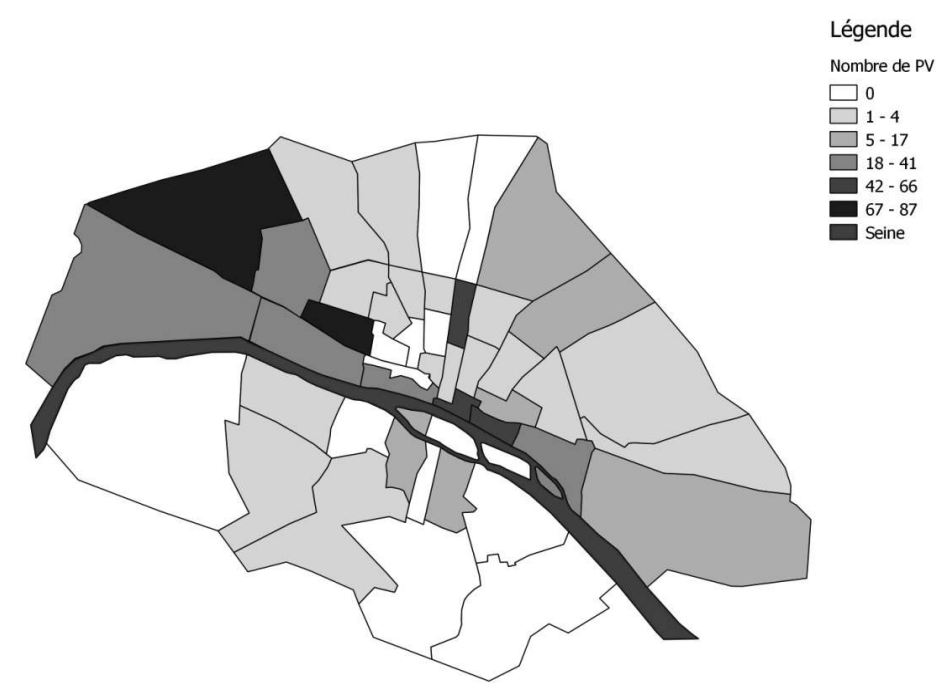

Figure 7. Carte 1 : Densité des procès-verbaux

La carte est difficilement exploitable en l'état à cause de l'inégalité de conservation des documents des sections. Elle ne permet pas d'établir une corrélation certaine entre la fréquence des délits et la géographie parisienne d'autant que de façon logique l'on pourrait supposer que les quartiers du centre de Paris où se concentrent les cochers et les marchés sont les espaces qui devraient être les plus foncés ${ }^{14}$. 


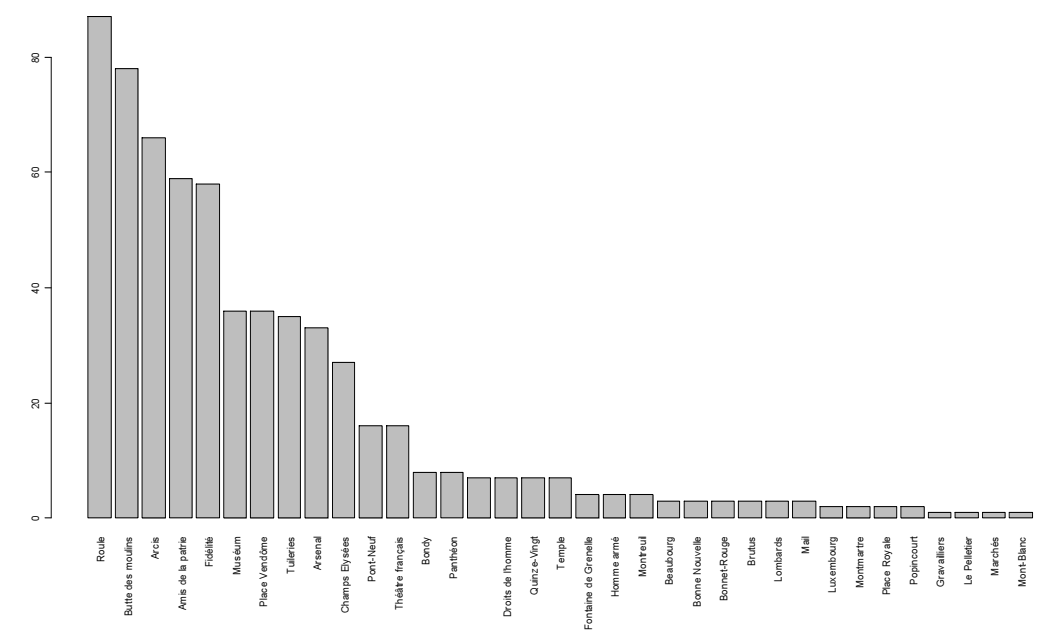

Figure 8. : Distribution des procès-verbaux selon les sections.

La remarque précédente est corroborée par cette répartition des procès-verbaux par sections. La géographie demeure aléatoire mais permet d'imaginer une partie perdue à jamais des traces. C'est dans les sections où les documents ont été les mieux conservés, celles de la Butte des Moulins, du Roule, des Arcis, de la Fidélité, que se trouvent le plus de cas ${ }^{15}$. Il faut désormais lire les procès-verbaux pour analyser plus à fond le métier du policier, ses moyens d'investiguer, et sa façon de régler les problèmes que lui posent les animaux et leurs maîtres.

\section{La police et la bête.}

\section{Le temps de la Révolution et le cheval, de la distinction nobiliaire à la comédie bourgeoise.}

Les quatre temps d'activité autour des animaux révèlent trois moments bien différents et tout à la fois caractéristiques. Le premier regroupe donc une attention moyenne du garant de l'ordre et renvoie le plus souvent, dans le détail des lectures des dossiers afférant aux périodes 1790-1792 et 1795-1797, à des affaires d'accident où le cheval, son cavalier ou son cocher jouent un rôle majeur. Quelques exemples suffisent à illustrer 
des situations typiques et leurs enjeux sociétaux presque toujours rejoués lors de chaque choc ou chute, parmi les 240 mentions concernant les chevaux en en faisant l'animal le plus surveillé. Chevaux abandonnés, chevaux perdus, carcasses de chevaux abandonnées, chevaux fauteurs de trouble lorsqu'on les mène à l'abattoir, chevaux périssant sous la charge et bloquant les rues, chevaux volés, chevaux atteints de morve et laissés à l'abandon, constituent la routine du policier scrupuleux. Autant de faits qui ne font que confirmer ce que l'infatigable marcheur dans Paris que fut Mercier dénonce souvent : la peur réelle du piéton parisien de se faire renverser à chaque coin de rue dans une ville sans trottoir, dans une ville où les coursiers sont lancés à toute allure ${ }^{16}$. Ainsi, plus intéressantes pour l'historien du politique, sont les situations conflictuelles qui disent des positions sociales tendues soudainement mises au jour par l'accident. Le 6 brumaire an II comparaît un particulier qui a renversé une femme avec son cheval, sans avoir fait mine de le détourner, alors qu'il avait lâché les rênes et ajustait son manteau de pluie. Il est amené devant le commissaire avec deux témoins. La jeune femme est « visitée » par un chirurgien qui décrit ses contusions à l'épaule et au genou gauche, aux reins, aux coudes. Elle est seule car son mari est aux armées. Elle a un enfant de huit ans et son état de gazière ne lui procure point assez pour survivre. De plus, elle est désormais hors d'état de pouvoir travailler. Dans le procès-verbal, le chirurgien fixe le dommage à 18 livres, ce que le citoyen Terrois, le cavalier, paie aussitôt, en plus des 10 livres d'amende dont il doit s'acquitter pour avoir lâché les brides de son cheval ${ }^{17}$. La même situation se répète plusieurs fois tout au long de la période, parfois de façon plus tragique. Le 27 prairial an VI, section de Montreuil, le commissaire Depatie se transporte rue de Charonne pour constater la mort d'une enfant de dix-sept mois qui vient d'être écrasée par une voiture qui ne s'est point arrêtée. La mère sous le choc ne peut répondre au commissaire. Témoin, le boulanger en face de la rue décrit l'accident ${ }^{18}$. Les postures ne sont pas caricaturales : ce ne sont point de grands seigneurs qui renversent avec mépris les manants. Elles n'en demeurent pas moins tout à fait explicites de rapport de force dont à la fin pâtissent toujours les plus démunis, parce que les moins capables de se protéger, obligés d'être dans la rue pour gagner leur vie. Plusieurs fois

(16) L'angoisse de MERCIER pour les chevaux lancés à bride abattue ou les roues de carrosses frôlant les parisiens est constante, parmi tant d'autres exemples « XX le bourgeois » ou « XXXIX Gare ! Gare ! » dans Tableau de Paris, tome I, réed. Mercure de France, Paris, 1994, p. 62, p. 108.

(17) Archives de la Préfecture de Police (désormais APP), et AA 69, Arsenal, affaire du 6 brumaire an II.

(18) APP, AA 173, Montreuil, affaire du 27 prairial an VI, folio 281. 
le commissaire joue un rôle médiateur, tançant le cavalier et l'obligeant à payer les soins et le préjudice fait à la personne clairement défavorisée socialement. Le lecteur perçoit le rôle pacificateur du commissaire et comprend bien la raison sociale qui s'échange dans la transaction organisée par le commissaire. La somme sera-t-elle suffisante ? Couvrira-t-elle toutes les séquelles ? Elle achète pour un temps le calme social. Entre les deux, le cavalier et l'accidenté, le cheval poursuit sa carrière de vecteur social des plus puissants. Du début à la fin de la Révolution, il conserve sa puissance de distinction. Dans cette société, et malgré le 26 août 1789 qui proclame l'égalité entre tous, le plafond de verre est concrètement la hauteur de l'encolure de l'animal que certains montent ou arborent et dont d'autres se garent ou regardent avec anxiété pour leur santé. Une autre source policière, plus politique, au-dessus des sections, celle du bureau de police du canton de Paris, né sous le Directoire, ne manque pas de le souligner. En l'an IV, le 3 messidor, à la rubrique Esprit public, il est noté que « le luxe qui s'étale dans les promenades publiques, les voitures et les cabriolets, les chevaux de luxe, excitent aussi les murmures de la misère et donnent lieu à beaucoup de propos $»^{19}$. À la fin du mois, on compte jusqu'à 1500 chevaux de luxe sur la route du bois de Boulogne ainsi qu'une grande quantité de voitures. « Cette magnificence a fait dire que c'était un fait exprès pour révolter le peuple de misère et porter les soldats à seconder une insurrection $»^{20}$. Un an plus tard, en germinal an V, le reproche est le même à Longchamp. «On entendait des sarcasmes sur la fortune subite et le luxe des nouveaux riches ; on a donné à quelques équipages des huées sourdes $»^{21}$. Le cheval devient le symptôme d'un malaise social qu'il cristallise plus que tout autre signe de distinction, animal choyé de la noblesse d'Ancien Régime, il redevient le symbole préféré d'une élite douteuse aux yeux de ses contemporains, parce que nouvelle, parvenue et réduite à l'imitation gauche de l'ancienne aristocratie. Le cheval est le mauvais signe d'une distinction sociale qui ne peut qu'irriter le peuple et inquiéter le policier jusqu'à ce qu'il reprenne les propos d'un journal : « les forgerons, les charpentiers, les selliers travaillent sans cesse à confectionner, à équiper les chars et les chevaux qui doivent traîner cette foule élégante et badine. Gloire à Longchamp, aux niais qui galopent, aux badauds qui les considèrent ! Ils font travailler, ils font vivre

(19) Alphonse Aulard, Paris pendant la réaction thermidorienne et sous le Directoire, Tome III, an IV, Paris, L. Cerf, 1899, p. 261.

(20) Ibidem, p 318.

(21) Ibid., Tome IV, p. 58, rapport du bureau central du 24 germinal an V. 
le pauvre monde $»^{22}$. La cité des abeilles de Mandeville où le luxe des uns donne du travail aux autres, semble transposée dans un Directoire dont l'image de régime corrompu commence dès son existence même à se construire, au travers du vice de la richesse de Paris transformé en cité des chevaux.

\section{La viande, la boucherie et la Terreur.}

La deuxième figure paradoxale de cette enquête sur la place de l'animal dans le quotidien du policier en temps de Révolution, est une figure d'animal mort sous la forme du quartier de viande. Si le cheval est l'animal le plus présent sur l'ensemble de la période, la question de la boucherie mobilise les policiers de toutes les sections ayant conservé quelques documents entre 1793 et 1794. Sont-ce vraiment des animaux dont il est question ici, ou d'autres formes animales ? Ce sont bien des animaux dont il est question ici car il ne faut pas oublier que le privilège du boucher est d'occire lui-même l'animal qu'il va ensuite préparer, ce qui implique que l'animal entre vivant dans Paris, et est perçu en tant que tel par les Parisiens. Une gravure, «La liberté des entrées », illustre la décision de suppression de l'octroi de Paris le 19 février 1791. De façon saisissante l'auteur, Meunier, a su rendre cette impression de troupeaux grouillant, chevaux de trait, chevaux de carrosses, grappe de bœufs désordonnés, moutons serviles, chiens aboyant, le tout traversant le pont situé à la barrière dite de « la Conférence » (à proximité de l'actuel pont d'Iéna).

Les bouchers ou leurs intermédiaires vont le plus souvent acheter la viande à Poissy puis ramènent les animaux sur pied qu'ils abattent dans leurs échaudoirs, offrant alors aux Parisiens un spectacle que Louis Sébastien Mercier a su rendre mieux que nul autre en décrivant les scènes de boucherie en pleine ville :

« Un jeune bœuf est terrassé, et sa tête armée est liée avec des cordes contre la terre ? Une lourde massue lui brise le crâne ; un large couteau lui fait au gosier une plaie profonde. Son sang qui fume coule à gros bouillon avec sa vie [...] des bras ensanglantés se plongent dans les entrailles fumantes; un soufflet gonfle l'animal expiré, et lui donne une forme 
hideuse. Ses membres partagés sous le couperet vont être distribués en morceaux, et l'animal est tout à la fois enseigne et marchandise $»^{23}$.

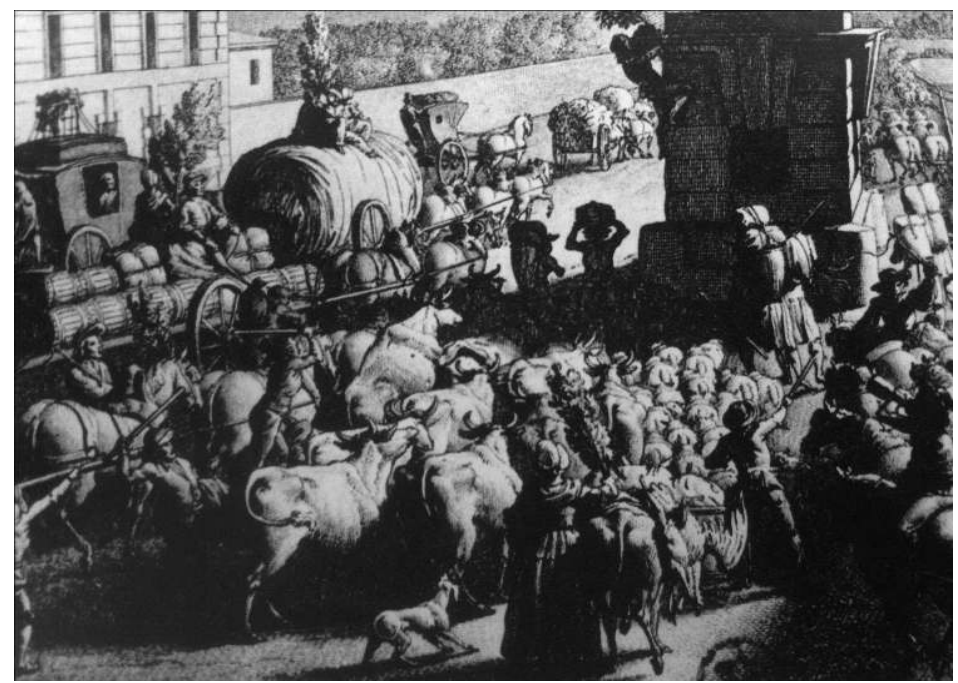

Figure 9. La liberté des entrées décrétée par l'Assemblée Nationale le 19 février1791, vue de la Barrière de la Conférence, par Meunier, Estampe, BNF, détail.

En 1793, ce n'est pas du tout cette sensibilité écorchée qui pose problème et qui explique subitement le pic de l'activité policière autour de la viande mais une conjonction de faits complexes qui vont en quelques mois dérégler entièrement la confrérie puis la profession de boucher ${ }^{24}$. Depuis 1776 et la réforme ratée de Turgot de la caisse de Poissy le métier est en crise. Entre une baisse relative de l'élevage, une croissance de la démographie parisienne, et un manque de liquidité des bouchers du aux conditions de l'achat des bêtes qui les poussent vers un endettement constant et des marges de bénéfice réduites, et ce malgré une montée des prix, la situation de la corporation se révèle difficile ${ }^{25}$. De plus, la question de l'hygiénisme de la ville divise les professionnels et les urbanistes entre

(23) Louis Sébastien MercIER, Tableau de Paris, chapitre XLII, « Boucheries », Paris, Mercure de France, réed., p. 112.

(24) Sydney WaTTS, Meat Matters, Butchers, Politics and Market Culture in Eighteenth Century Paris, University of Rocherster Press, 2006. 1908.

(25) Cf Henri Bourgin, L'Industrie de la boucherie à Paris pendant la Révolution, Paris 
eux. Dans le plan d'assainissement des villes certains désirent éloigner de la ville les tueries, pendant que d'autres, tentent de démontrer que ce qui aurait été gagné d'un côté en terme de propreté urbaine, serait perdu d'un autre côté, en terme de fraîcheur de viande proposée aux Parisiens. Tous n'ont que les expressions du bien public, de l'utilité publique au bout de la plume ${ }^{26}$. Dans ces conditions, le début de la Révolution opère des bouleversements sans précédent qui désorganisent le métier de boucher à plusieurs niveaux. Malgré les protestations des maîtres et syndics de la profession, la corporation est dissoute comme toutes les autres au printemps $1791^{27}$. Désormais la déréglementation du métier voit la croissance des petits étaux, et leur dispersion dans Paris, alors qu'auparavant, ils étaient concentrés de façon précise dans des marchés surveillés par la police tant pour le prix que pour la qualité des produits vendus. Dans un second temps, le métier qui concentrait les activités, de l'achat, du transport, de l'abattage puis de la vente, quatre opérations qui enorgueillissent le boucher et lui confèrent son savoir-faire, se défait et les activités se dispersent également en une division des tâches qui rend la traçabilité des produits difficile en cas de litiges toujours plus fréquents pour cause d'une viande avariée. En supprimant la corporation, la loi de l'Assemblée constituante, a laissé s'implanter un libéralisme des plus sauvages, où, nul ne s'enrichissant, nombreux sont ceux qui sont prêts à vendre n'importe quelle viande de n'importe quelle qualité pour survivre. Ceux-là existaient auparavant, regrattiers, revendeurs, «bouchers hors système » comme les appelle Bourgin, ils étaient surveillés et punis. Ces mercandiers de plus en plus nombreux offrent une viande douteuse que la commune de Paris malgré ces efforts constants ne peut endiguer ${ }^{28}$. Cette désorganisation de la boucherie parisienne se trouve renforcée par la conjoncture de crise économique dans laquelle la guerre extérieure puis la guerre civile plongent la France entre 1793 et 1794. L'inflation de l'assignat, l'argent métallique qui se cache, la pénurie de bêtes, l'économie dirigée d'un côté et le marché noir de l'autre, l'augmentation de la précarité des ouvriers parisiens, font du quartier de viande une denrée rare et subitement sous le regard de la police. Comme le signalait Albert Mathiez, "dans un monde qui a faim », rendu sensible par la pénibilité des travaux de force dans le Paris de guerre, rendu plus

(26) Mémoires concernant les boucheries de Paris, pour les syndics, adjoints en charge député et communautés des maitres bouchers de Paris, Paris, mars 1790, chez Gueffier.

(27) Voir la carte des boucheries du XV au XVIII ${ }^{\mathrm{e}}$ siècle proposée par L. Mirlou et B. Descamps, http://alpage.huma-num.fr/documents/ressources/cartes/boucheries_rang.pdf, consulté le 3 mars 2014.

(28) Ct . Henr1 BOURGIN, op. cit. p 41-42. 
souffreteux par la pollution des usines de guerre installées partout où cela est possible dans la capitale, angoissé par les nouvelles du front et le climat anxiogène des jugements politiques et des luttes meurtrières des factions dont l'écho est fort dans les sections, la question de la viande de boucherie et de la consommation des animaux devient centrale dans le métier policier, comme l'attestent les archives ${ }^{29}$.

Il faut d'abord traquer les mercandiers qui vendent une viande douteuse souvent avariée. Ainsi le 23 vendémiaire an II (14 octobre 1793), un citoyen déclare avoir vu passer une particulière avec une hotte remplie de viande. D'autres citoyens constatent que cette viande «suffoquait». Le commissaire intervient, confisque la hotte de Marie Drouilly, âgée de quarante cinq ans, et la fait transporter chez un équarisseur ${ }^{30}$. Il faut ensuite repérer les ventes clandestines opérées de maison en maison. Le 21 messidor an II, le commissaire de la section de l'Arsenal est prévenu d'une fraude opérée par deux citoyennes qui vendent de la viande à un prix excédant le maximum, et notamment le mouton à 24 sols la livre, se présentant de maison en maison. Une des deux citoyennes, Suzanne Andut, veuve Satieu, qui vend des herbages dans les rues, est arrêtée. Le policier lui explique son infraction : seule l'administration des subsistances a le droit de fixer les prix ; il est permis au citoyen de s'approvisionner mais jamais de survendre la denrée. De façon classique, la citoyenne prétend s'être retrouvée avec trop de viande pour elle et avoir tenté de vendre le surplus. Pourtant une autre citoyenne vient témoigner en affirmant qu'elle l'avait bien prévenue « qu'elle vendait au mépris des lois sur le maximum et qu'elle doit savoir que les règlements à cet égard sont très sévères $»^{31}$. Il faut surveiller aussi les bouchers clandestins. Le 6 germinal an II, section Fidélité, la citoyenne Marie-Jeanne Charlotte a déclaré qu'un nommé Henriot demeurant rue de la Mortellerie, au troisième étage de son immeuble, vendait de la viande en cachette 24 sols pour le veau et 20 sols pour le bœuf. L'enquête commence où le commissaire apprend d'une autre citoyenne qu' un boucher est sorti de la dite maison avec un quartier de bœuf. Plusieurs citoyens attendent même au bas de la maison pour être servis. Le commissaire se rend sur place, fait ouvrir la pièce et découvre un billot de bois pour couper la viande, un morceau de viande et les restes de nombreux os. L'épouse prétend que son mari, étalier rue de Charenton, a reçu cette viande du citoyen Armand, boucher, et nie toute transaction de viande. Le commissaire fait arrêter

(29) Albert MATHIEZ, La vie chère et le mouvement social sous la Terreur, Paris, Payot, 1927.

(30) APP, Section des Arcis, AA 60, affaire du 23 vendémiaire an II.

(31) APP, Section de l'Arsenal, affaire du 21 messidor an II. 
le mari, saisit la viande, la transporte chez le citoyen Langlois, boucher, afin de la distribuer aux malades et aux indigents à petits lots, au prix du maximum $^{32}$.

Il faut ensuite surveiller la vente de viande avariée par les bouchers eux-mêmes. En ces temps de pénurie, les problèmes de viande gâtée se posent également sur l'étal du boucher. Section de l'Arsenal, le 12 vendémiaire an II (3 octobre 1793), le commissaire Camoin est sollicité par la citoyenne Corroye déclarant avoir acheté au citoyen Pion, boucher, rue Saint Paul, une quantité de cinq livres de viande au prix de 17 sols la livres dix livres. Ce n'est qu' arrivée chez elle qu' elle découvrit un morceau avarié. De retour ${ }^{33}$ chez le boucher, celui-ci refuse de le remplacer. Elle a trois enfants et sa sœur à charge et c'est tout ce qu'elle possède pour la semaine. Le commissaire se déplace pour tenter la transaction « attendu que la surveillance du comestible nous est comptée », transaction refusée par le boucher et sa femme qui tiennent tête au représentant de l'ordre. Penaud, le commissaire ne peut que rendre le morceau de viande à la citoyenne et prévient le boucher qu'il va faire son devoir ; ce qu'il fait en rédigeant un rapport dans lequel il conclut « qu'il est essentiel de réprimer la vexation de la part des marchands, seul moyen capable de faire naître les troubles dans cette ville ». Fort nombreuses sont pareillement les affaires où le commissaire est pris à témoin par les Parisiens de la mauvaise volonté des bouchers à pratiquer le prix imposé par la loi sur les denrées de première nécessité dont la viande. Ainsi le contrôle du respect du maximum accapare les commissaires et leurs agents pendant la période d'économie dirigée et avec eux leur corollaire logique, la gestion de la pénurie de viande, la traque du marché clandestin, la tentative permanente de déjouer les tensions sociales risquant de provoquer des colères politiques. Ils sont aidés pour cela par la mise en place de la Boucherie générale de Paris, à la tête de laquelle est placé le citoyen Sauvegrain, sûrement par Lindet, spécialiste des subsistances au Comité de salut public ${ }^{34}$. Le Comité de salut public prend ainsi la décision par son arrêté du 8 germinal an II (28 mars 1794) de confier à la municipalité l'administration des subsistances, de recevoir et de se charger des bestiaux, de les transmettre et les distribuer sur le champ

(32) APP, Section Fidélité, AA 139, affaire du 6 germinal an II.

(33) APP, Section de l'Arsenal, AA 69, affaire du 12 vendémiaire an II.

(34) Cf Éric Szulman, De l'étal à l'écriture, Jean Baptiste Sauvegrain (1753-1829), mémoire de maîtrise sous la direction de Dominique MARGAIRAZ et Denis WorONOFF, 1999-2000, p. 37. Sauvegrain dans ses observations de 1815 confie qu'il fut chargé pendant les 28 mois de son office de faire abattre chaque jour 110 à 120 bœufs, 4 à 500 veaux, 4 à 500 moutons et de faire fondre tous les suifs nécessaires à cet abattage considérable» p. 9 . 
aux bouchers et charcutiers. Ces derniers, avec les traiteurs, restaurateurs et aubergistes de Paris ne peuvent s'approvisionner d'animaux qu'auprès de l'administration des subsistances de la commune ${ }^{35}$. À partir de cette date la liberté du commerce de la viande est suspendue pour une durée illimitée. Mais rien n'y fit : la pénurie de viande enflait, malgré les efforts des officiers municipaux et de Sauvegrain, malgré la carte de rationnement instituée depuis le 29 octobre 1793, malgré l'invention d'un carême civique afin de réduire la consommation de viande. Le prix de la livre fixé entre 14 et 15 sous la livre fut souvent dépassé et intenable pour les bouchers qui fermaient ${ }^{36}$. Quelques mois plus tard après la crise de Thermidor, la situation du marché de la viande est toujours aussi tendue mais la politique économique a changé.

L'an III voit encore les policiers mobilisés sur la question de la viande et de la boucherie. Cette fois, c'est la déstructuration brutale du maximum et les rigueurs d'un des deux hivers les plus froids du siècle, qui provoquent une rapide paupérisation de la population parisienne réduite pour une partie d'entre elle au dénuement absolu ${ }^{37}$. Il faut encore en l'an III, période de grande détresse sociale et de misère continue, continuer de déjouer les ruses des vendeurs clandestins. Ainsi le 23 germinal, le commissaire de la section des amis de la Patrie reçoit un rapport de ses agents qui lui signale que l'on vend Porte Denis de la viande de très mauvaise qualité. Le commissaire donne l'ordre d'enquêter et ce qui est important de se faire aider de deux bouchers pour vérifier la qualité de la viande ; en fait les marchands se tiennent constamment à la limite de la section des Amis de la Patrie et de la section Bonne Nouvelle, déjouant les visites policières ${ }^{38}$. Les journées de Germinal puis de Prairial restent le fruit de la misère et de la colère du dérèglement de la société, à la suite de l'abolition du maximum, de l'hiver sans égal et de la claire volonté des gouvernants de ne plus placer la question sociale au cœur du politique. L'arrêté du Comité de salut public du 27 germinal an III (16 avril 1795) énonce qu'à partir du 21 floréal suivant, les bouchers chargés de distribuer la viande destinée par le gouvernement à la consommation des habitants de Paris seront et demeureront supprimés, et pourront faire le commerce pour leur compte. L'article deux stipule que la section des subsistances générales nommera

(35) AN, AFII 68.

(36) Éric SZULMAN, op. cit., p 37-38.

(37) Richard СовB, La Mort est dans Paris - Enquête sur le suicide, le meurtre et autres morts subites à Paris, au lendemain de la Terreur, octobre 1795-septembre 1801, Paris, Le chemin vert, 1985.

(38) APP, AA 50, Amis de la Patrie, affaire du 25 germinal an III 
sur la présentation des comités civils et de bienfaisance des sections, des bouchers au nombre de 5 par section pour continuer la distribution aux citoyens de Paris. L'étau de famine se resserre davantage sur les catégories les plus démunies de la capitale. Une affaire livre le résumé de tant d'autres. Le 13 floréal an III, deux commissaires du comité de bienfaisance de la section viennent se plaindre d'un dénommé Denont, gendarme. Sa famille n'a point eu de viande depuis trois jours car les commissaires repoussaient les femmes. Il allait donc se plaindre directement au Comité de sûreté générale. Denont, convoqué par le commissaire, raconte que, la veille, son épouse s'est présentée chez le boucher mais la distribution de viande faite, on lui a refusé son numéro, et qu'ayant entendu battre la caisse, lui-même s'est présenté au comité de bienfaisance pour recevoir un numéro. On lui répond qu'il faut faire comme tout le monde, se présenter à trois heures du matin s'il voulait en avoir. Sa femme journalière et lui ayant un bras invalide, elle ne peut perdre trois jours de travail par décade pour recevoir la viande de la famille ${ }^{39}$. Elle ne peut attendre des heures sans mettre en péril le foyer, et elle ne peut assez gagner d'argent pour payer la viande sur le marché sans aide du comité. Le gendarme en tire la conclusion «qu'on le pousse à se mettre voleur pour pouvoir exister ». Après une provocation, le gendarme tente de sauver son honneur mais c'est finalement lui qui est inquiété pour insulte à agent public ${ }^{40}$. L'enjeu est économique, policier mais surtout devient politique. Encore quelques mois et un arrêté de pluviôse an IV stoppe la distribution de viande aux citoyens. Le 12 pluviôse an IV, l'arrêté du Directoire exécutif fait reposer la responsabilité de la crise sur les « effets naturels de la loi sur le maximum ; constate que le commerce reprend son cours, et se trouve dans un état tranquillisant pour les citoyens »; il faut donc constater que la distribution se fait à trop bas prix et nuit au rétablissement des finances de la République ; en conséquence, l'article Ier énonce : « la distribution de pain et de viande faite par le Gouvernement aux habitants de Paris cessera à compter du Ier ventôse prochain $»^{41}$. Comme dans le cas du cheval, l'animal sous la forme du quartier de viande a été un enjeu de construction d'une politique de claire hiérarchisation des groupes sociaux.

(39) Sur le rôle des femmes dans la prise de conscience sociale et politique du peuple de Paris en l'an III, voir Dominique GodineaU, Citoyennes tricoteuses, Paris, Perrin 2004.

(40) APP, AA 51, Amis de la Patrie, affaire du 13 floréal an III.

(41) Cité par Éric Szulman, De l'étal à l'écriture..., op. cit., p. 43. 


\section{Le Directoire ou compter les vaches et les bœufs en ville.}

Il existe un second pic d'interventions policières dans le graphique pluriannuel. Il concerne particulièrement une enquête systématiquement organisée en brumaire en IV, en vue de l'éradication d'une épizootie, manifestement passé inaperçue des historiens de Paris mais que la lecture exhaustive des documents fait apparaître le plus clairement. Entre brumaire et frimaire an VI (novembre-décembre 1797), la quasi-totalité des commissaires dont les documents ont été conservés, vont devoir successivement organiser la visite des nourriciers, ou bouveries, afin de laisser la possibilité aux élèves vétérinaires de l'école de Maisons-Alfort de pouvoir vérifier leur état de santé et les déclarer hors de danger ou à supprimer dans les plus brefs délais. Ainsi les divisions de Popincourt, Pont-Neuf, Place Vendôme, Panthéon, Museum, Fontaine de Grenelle, Butte des Moulins, Amis de la Patrie, des Arcis, de Beaubourg, du Bonnet Rouge, de Gravilliers, du Mail, du Marché, du Montblanc, de Montreuil, du Temple, des Quinze-Vingt, du Théâtre français, connaissent en l'espace d'un mois, la visite conjointe des mêmes experts vétérinaires mandatés par le bureau du canton de Police de Paris. L'enquête se poursuit au-delà de frimaire jusqu'en germinal (mars 1798), preuve des inquiétudes de reprise de la maladie et de la mobilisation des officiers de santé et de police. Le commissaire de la section place Vendôme, par exemple, reçoit un imprimé dans lequel l'administration centrale du département lui ordonne d'accompagner les experts vétérinaires pour contrôler les vaches laitières. Ils doivent se faire ouvrir les étables au besoin en étant secondés par la force armée. Le lecteur retrouve les mêmes noms d'experts, se présentant avant chaque visite aux commissaires, pour se faire accompagner dans les étables de leur connaissance. Le résultat produit des enquêtes plus ou moins bien menées, et lisibles. L'ère de la statistique naissante n'ayant pas encore transformé les mœurs administratives des policiers ${ }^{42}$.

C'est pourtant la seule enquête transversale de la décennie dans ce domaine. Elle prouve la volonté des autorités en charge de la ville de Paris de veiller à la santé publique en surveillant des risques d'épizootie, dans une politique volontaire liant différents corps de l'État, vétérinaires et policiers. Sevestre, Rochet Le Prévost, Dieuzeide, Carville, Guigny, Le Rouge, Quentin (du Loir-et-Cher ou de la Meuse) sont tous des élèves

(42) Jean-Claude PERrot, L'âge d'or de la statistique régionale française an IV-1804, Paris, Ser, 1977, voir aussi François VALLAT, Les Bæufs malades de la peste, la peste bovine en France et en Europe, XVIII-XIX $X^{e}$ siècle, Rennes, PUR, 2009. 
vétérinaires qui sont entrés à l'école en l'an III et sont donc opérationnels pour traiter les épizooties en milieu urbain. Ils savent reconnaître les cas de bêtes infectées, à traiter en priorité, dans le protocole invariable de la visite sanitaire qu'ils opérèrent de division en division ${ }^{43}$. Le matin de bonne heure, les experts, par deux, se présentent chez le policier qui commence la tournée des étables. Les connaît-il toutes ? Sont-elles toutes réellement visitées ? En l'état il est difficile de répondre et les résultats quantifiés sont des plus délicats à manier. Entre la visite aux Gravilliers dans la seconde vague de visite le 16 germinal dont le rapport se termine par un laconique «nous nous sommes transportés chez tous les citoyens qui ont des vaches ; après les avoir trouvées très saine, examinées par le dit Prévost », et l'enquête du commissaire de la division du Bonnet Rouge qui offre un tableau détaillé, l'écart de qualité est trop important pour pouvoir conclure sur le nombre réel de vaches dans Paris et leur contamination réelle. Division du Bonnet Rouge justement, la visite des bêtes à corne a été menée par les enquêteurs vétérinaires Carville et Lerouge dans 43 endroits différents, en deux jours et deux fois six heures de $10 \mathrm{~h}$ à 16 heures. Au cours de cette recherche de la vache malade, ils observent 169 vaches, dont une est morte et envoyée à l'équarisseur, 5 malades à tuer. La lecture du rapport montre surtout une grande dispersion des lieux de garde des ruminants. Les gardiens ou gardiennes peuvent en posséder un seul, là où un dénommé Dreux en possède $16^{44}$. La plupart en ont moins de cinq, confirmant la dispersion des métiers de la boucherie et la difficulté de contrôle, plainte qui commence à se structurer de plus en plus durant ces années ${ }^{45}$. Division Popincourt le 25 brumaire an VI, le commissaire, sans donner le détail des adresses et des noms de gardiennes et gardiens d'étable, fait état de 18 endroits visités pour 259 bêtes « dont aucune n'est atteinte par la maladie... exceptées 9 médicamentées et pansées par deux élèves de l'École vétérinaire $»^{46}$. Les visites ne se passent pas toujours sereinement. Tel nourrisseur, division du Mail, qui a trois vaches, refuse de les faire palper par le vétérinaire arguant que c'est le médecin lui-même qui risquerait de porter la contagion dans son étable s'il n'avait pas changé ses vêtements ou s'il ne s'était point

(43) Je remercie Malik Mellah qui m'a aidé à identifier ces experts, qui font partie de son corpus de thèse sur l'École vétérinaire de Maisons-Alfort.

(44) La lecture de l'enquête de la division des Arcis ressemble à ce premier constat. Dans le cœur de Paris, 11 lieux sont visités avec souvent un seul animal inventorié, seul le citoyen Fleury rue de la Potterie possède 8 vaches. En tout l'enquête révèle 35 bêtes à cornes, bœufs et vaches confondus, AA 63,15 brumaire an VI.

(45) APP, AA 78, Bonnet rouge, affaire du 29 brumaire an VI.

(46) APP, AA 219, affaire du 25 brumaire an VI. 
lavé au vinaigre. Finalement le commissaire écrit qu'il va surveiller les deux étables de sa division, donc à l'effectif bien plus faible, en prévenant les autorités supérieures en cas de maladie. En fin de rapport, il écrit : « cette contagion parait avoir été portée dans les bestiaux de ce pays par les vaches venant de la Flandre $»^{47}$. La situation ne doit pas s'améliorer tout de suite, au contraire, une nouvelle vague de visite est commanditée en germinal an VI sous l'égide du bureau de police. L'administration centrale du département de la Seine informe que la maladie épizootique sur les bêtes à cornes, loin de diminuer, augmente chaque jour. Elle recommande par une lettre du 24 germinal an VII, l'exécution de ses arrêtés des 7 et 24 brumaire VI et 14 frimaire an VI, qui ont donné lieu à un premier recensement. «Comme depuis la dernière visite des vaches laitières, il en est entré beaucoup dans Paris, nous avons cru devoir ordonner une nouvelle visite chez tous les nourrisseurs et autres propriétaires de vaches dans l'étendue de cette commune ${ }^{48}$. Gobert, le commissaire de la division des Quinze-Vingts, qui avait déjà mené son enquête en brumaire, est le seul qui décrit « les sinthomes » (sic) de la maladie, soit « une inflammation générale » des mamelles qui se traduit par un mélange de sang et de lait au moment de les traire. C'est son successeur, Lebanc, qui reprend l'enquête avec les instructions reçues au début de floréal. Le premier jour, il a visité 214 vaches appartenant à 18 vachers. Le lendemain il se transporte chez 16 vachers qui lui exposent 234 vaches, « aucunement attaquées de la maladie épizootique $»^{49}$. L'absence de relance de visite de la part du bureau central du canton de Paris, laisse supposer un moins grand danger et une décrue de la maladie. Deux fois en brumaire-frimaire an VI puis en germinal-floréal an VII, les commissaires se sont mis au service d'une police sanitaire démontrant un plus grand soin de la part des autorités de dépister tout danger d'épizootie dans la capitale. De façon générale, il faut reconnaître que depuis le début du Directoire, le contrôle des bouchers et surtout de l'assainissement et de l'hygiène se fait davantage ressentir. Les simples citoyens semblent désormais davantage sensibles à toutes les formes de pollution liées ou dérivées de la boucherie. De fait, les enquêtes ne sont pas forcément nouvelles mais elles indiquent clairement l'attention soutenue

(47) APP, AA 169, Section du Mail, brumaire an VI non daté.

(48) APP, AA 211, Place Vendôme, 24 germinal an VI.

(49) APP, AA 221, Quinze-Vingt, affaire du 2 floréal an VI. 
de la police, davantage consciente de son service au public, concernant la propreté liée à la question du sang ${ }^{50}$.

Le bureau de salubrité du bureau central du canton de Paris envoie ainsi le 26 floréal an IV un imprimé à tous les commissaires, pour les engager expressément à surveiller les bouchers qui laissent couler dans le ruisseau le sang des animaux qu'ils abattent ${ }^{51}$. L'attention se porte également sur la nature de la viande. La question tourne autour de la viande de cheval dont la corruption rapide empêche qu'on puisse la vendre dans la rue. Comment savoir de plus si l'animal n'est pas mort de la morve ou du sarin ? Pour cela, il faudrait conserver la tête, ce qui est impossible pour le vendeur. Il faut donc que la police interdise ces ventes pour accomplir « les devoirs de son ministère et de l'humanité » dit le formulaire afin de protéger ceux qui, tiraillés par la faim, pourraient acheter n'importe quoi ${ }^{52}$. Enfin, classiques mais répétées sont les visites que le commissaire effectue avec un architecte qui l'assiste dans une enquête de comodo/incomodo, lorsque le voisinage se plaint des activités liées à la boucherie et ses activités industrielles dérivées, le suif surtout. Ainsi le 7 messidor an VI une enquête est menée rue du Grand hurleur car il existe une fonderie de suif qu'il faut visiter pour constater sa conformité aux risques d'incendie. Manifestement les conditions ne sont pas remplies et les habitants redoutent le feu et les émanations nuisibles à leur santé. Moitte, l'architecte, doit dresser le constat nécessaire et conclut de façon négative quant à la viabilité de cette fonderie s'appuyant sur l'arrêté du 7 fructidor an IV $^{53}$. Division du Museum, le 18 vendémiaire an VI, la visite de l'échaudoir du citoyen Marcel, boucher et mercandier, révèle que son local ne peut réunir les conditions de l'arrêté du 13 vendémiaire an $\mathrm{V}$ quant aux tueries. L'échaudoir étant situé dans un immeuble de 115 locataires dont une grande quantité d'enfants, il ne doit pas les mettre en danger ${ }^{54}$. Procédurière et encadrée par des textes normatifs, la police s'invente un ordre républicain, continuant son ancienne fonction de surveillance des marchés, la garantissant au nom d'un nouvel ordre public conscient que la faim est source de colère sociale, conscient que la responsabilité républicaine entraîne un nouveau rapport à la population. Le policier est responsable des animaux morts qui s'échangent, par leur dimension économique, par la situation hygiénique, mais aussi parce que,

(50) Cf. Thomas Le RouX, Le laboratoire des pollutions industrielles, Paris, 1770-1830, Paris, Albin Michel, 2011.

(51) APP, AA 99, Butte des moulins, imprimé du 26 floréal an IV.

(52) APP, AA99, 14 germinal an IV, lettre de la division du commerce du bureau central.

(53) APP, AA 52, bureau de salubrité et voie publique, 7 messidor an VI.

(54) APP, AA 190, 18 vendémiaire an VI. 
les sensibilités se modifiant quelque peu, l'animal n'est pas n'importe quel spectacle : il est édifiant. Différent de l'homme, il peut supporter davantage et travailler pour lui, voire le nourrir ; être vivant comme lui, il lui renvoie une image confuse et déformée de lui-même qui peut être source de troubles et que le policier doit veiller à ne pas laisser se développer.

\section{En république, le spectacle de l'animal ne doit pas continuer.}

Le dernier point abordé est quantitativement insignifiant par rapport aux soucis que les chevaux, bœufs et vaches donnent aux policiers. Il n'en est pas moins révélateur du rôle civique que doit jouer la police, garante de l'ordre public, c'est-à-dire en fin de compte dire ce qui est licite sous la forme du visible dans la rue révolutionnée. Le spectacle urbain est une science politique au sens littéral du terme. La rue doit offrir, autant que faire se peut, la démonstration des valeurs qui fondent la république de la décence, de la vertu, de la retenue, de l'égalité, du refus des spectacles dégradants, du refus du spectacle qui détourne tout un chacun de son travail, du refus des spectacles qui rappellent le libertinage oisif de l'Ancien Régime ou ses mœurs brutales. Or le pavé de Paris, avant et après 1789, est recouvert de petits tréteaux où sont exhibés des thêâtres d'animaux savants, de chèvres lettrées, de chats musiciens à leurs queues défendant, d'ours muselés ou de chiens acrobates, sans même évoquer les grands succès de monstration d'animaux exotiques dans les foires de Saint Germain entre autres $^{55}$. Il est un lieu encore plus emblématique que les autres, dit «le combat du taureau » au nord de Paris, porte de Pantin.

(55) Cf. Almanach forain ou les différents spectacles des boulevards et des foires de Paris, à Paris, chez Valleyre, 1773. 


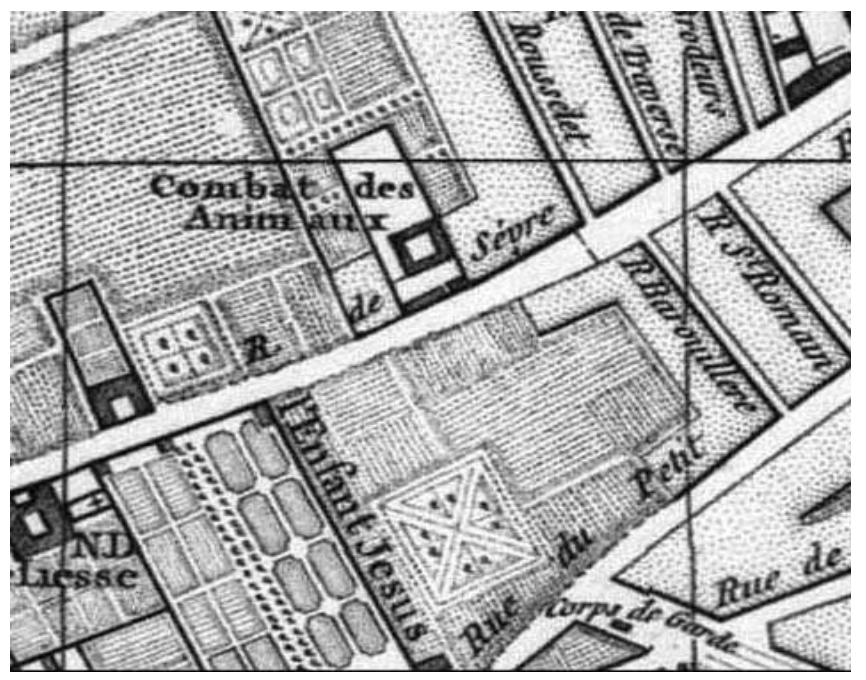

Figure 10. Le clos du Combat des animaux sur la carte de Vaugondy (1771) (voir Alpage pour plan Verniquet)

À partir de 1780, assez régulièrement dans la presse, des nouvelles de combats entre animaux sont données. En plus des articles du Journal de Paris d'août et novembre 1781, une annonce paraît le 23 avril 1781 dans Les Mémoires Secrets. L'auteur y relate une course de taureaux à l'espagnole où un taureau fut mis à mort par les «tauréodores », c'est-à-dire par les hommes combattant contre cet animal, alors que la police avait, il est vrai, interdit pareil spectacle ${ }^{56}$. Ainsi, l'habitude, avec une tolérance policière évidente, fut prise d'organiser ces sortes de combats le plus souvent engageant de solides bouledogues contre des taureaux, ou des chiens de combat entre eux. En 1788 on signale encore des combats de dogues contre différents animaux féroces tels que le sanglier, le loup, le léopard, le tigre, le lion, qui se terminent toujours par des feux d'artifice, sources le plus souvent de cruauté envers chiens et chats à la queue desquels on attache les pétards ${ }^{57}$. Quelle pouvait être l'attitude du législateur et fondateur de la nouvelle cité républicaine devant ce qui ne ressemblait que trop à la décadence de Rome et de ses anciens jeux de cirque, si ce n'est régénérer

(56) Mémoires secrets pour servir à l'histoire de la République des lettres en France depuis 1772 jusqu'à nos jours, ou journal d'un observateurs, tome 17, chez Adamson, 1782, p. 146-147, 23 avril 1781,

(57) M. THIERY, Le voyageur à Paris, extrait du guide des amateurs et des étrangers voyageurs à Paris, Paris, Hardouin et Gattey, 1788. 
les mœurs par tous les moyens et pour commencer, interdire le spectacle des animaux dressés, spectacle d'animaux esclaves, humiliant peut être davantage les citoyennes et citoyens avides de ces scènes que les bêtes concernées. De plus, les animaux exotiques seraient-ils des agents rampants ou sautants de la Contre Révolution ? Le 6 juillet 1790 une plainte est déposée au comité du District de Saint-Germain. Un négociant dénonce un complot ourdi par des aristocrates qui auraient caché dans des tentes, aux environs du Champ de Mars, plusieurs « animaux tels que lyon [sic], tigres et léopards (une note dans la marge ajoute hyène) $»^{58}$. « Les rumeurs assez fortes » prétendent que les animaux seront lâchés sur la foule pour « faire tuer plusieurs citoyens ». Deux autres citoyens, Antoine Marquis et un dénommé Rosswag, ont déclaré avoir vu autour du Champ de Mars, des animaux féroces qui seront « utiles aux aristocrates pour donner lieu à des massacres ». La décision de police est de faire tuer tous les animaux féroces qui sont dans Paris, et notamment ceux qui sont exposés aux environs du Champ de Mars. La rumeur, car manifestement c'en fut une, ne semble pas avoir laissé d'autre souvenir. Pourtant le récit est intéressant qui pose d'un côté les citoyens, de l'autre les aristocrates, avec entre les deux des armes féroces, les bêtes fauves. Ce qui était le signe de la distinction de la mode exotique sous l'Ancien Régime devient l'outil de la haine politique et homicide. Les animaux se retrouvent instrumentalisés et deviennent des enjeux politiques. Le plus vraisemblable est que l'émigration nobiliaire, parfois organisée dans la précipitation, a dû conduire à l'abandon d'animaux dangereux. Pour autant les spectacles d'animaux continuent, les métaphores animales pour désigner l'autre, deviennent monnaie courante intégrant le monde imaginaire de l'animal à celui des bêtes de pouvoir, jusqu'à ce que la république naissante, dans la foule des chantiers ouverts pour républicaniser le pays, s'attaque au spectacle des rues. La commune de Paris prend la décision le 13 brumaire an II (3 novembre 1793) de saisir les animaux vivants que l'on fait voir aux badauds tout en indemnisant les propriétaires ${ }^{59}$.

(58) APP, AA 179, affaire du 6 juillet 1790.

(59) Voir le récit qu'en font Yves LAISSUS et Jean-Jacques PETTER dans Les Animaux du Museum 1793 1993, Paris, Imprimerie nationale, 1993, p. 83. 

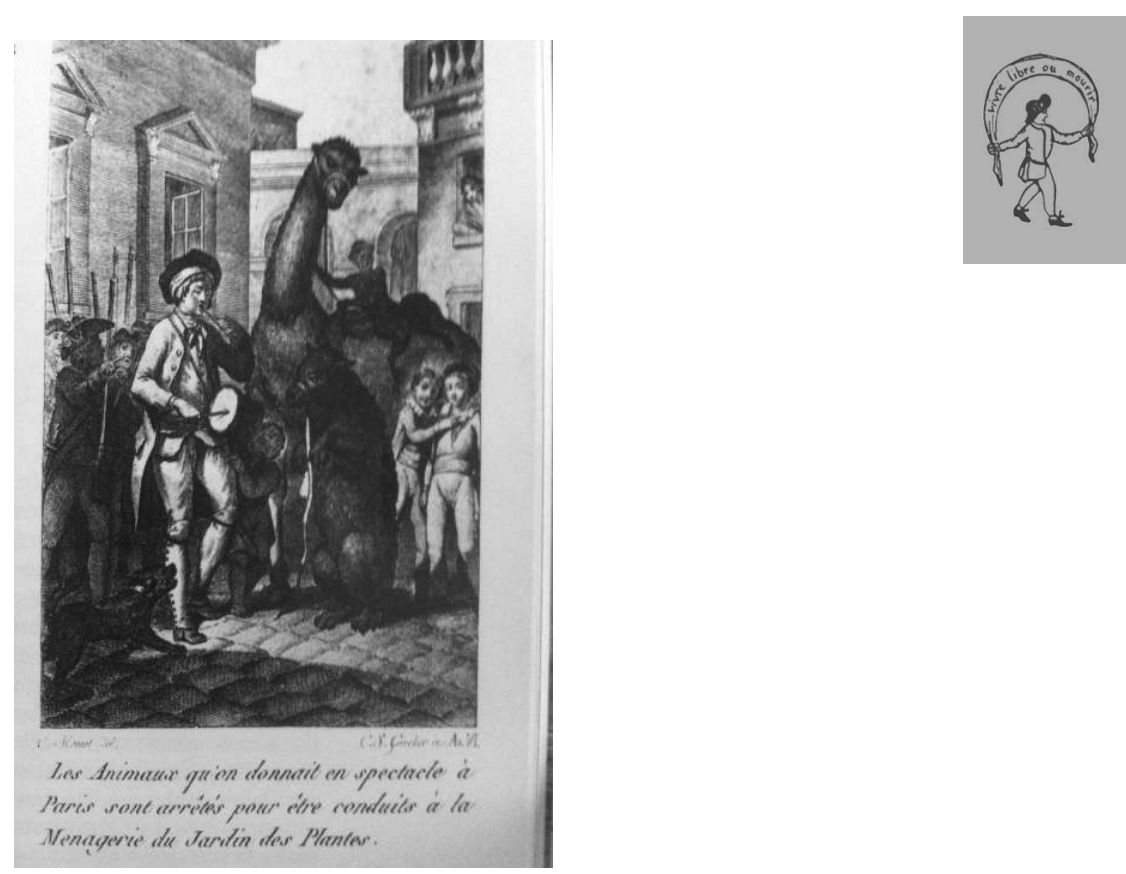

Figure 11. Gravure extraite du Voyage au jardin des plantes par L. F. Jauffret, ss d. (BNF)

Le 6 novembre, Desfontaines, le secrétaire du Museum, note qu'il est arrivé entre-temps un chat-tigre, un ours marin mâle, deux singes mandrills et trois aigles ${ }^{60}$. Un procès-verbal de la section des Arcis explique très concrètement l'opération de saisie des animaux. Deux officiers de paix se présentent chez le commissaire le 17 brumaire. Ils ont pour ordre de faire retirer de dessus les places publiques toutes les bêtes fauves que les charlatans font voir et les faire conduire au Muséum d'histoire naturelle, au Jardin des Plantes. Un dénommé Louzardi qui possède une baraque est interpellé. Il obéit et l'inventaire de ses animaux se fait devant Lucas, le concierge du Muséum. Un ours blanc, deux singes mandrills, trois aigles, à tête blanche, un chat tigre et deux agoutis sont laissés sur place, estimés ensemble à plus de 16000 livres avec les cages et la voiture qui les transporte. En floréal (le 27 de l'an II) l'arrivée régulière d'animaux saisis pousse le Comité de salut public à engager des frais et à transporter les

(60) Ibidem, p. 84. C'est manifestement la prise évoquée le 6 novembre par Desfontaines. Le secrétaire du Museum ajoute qu'il est arrivé entre temps un chat-tigre, un ours marin mâle, deux singes mandrills et trois aigles. 
grilles de la ménagerie de Versailles afin de construire de nouvelles cages. Exit de la rue donc, les animaux exhibés dans des conditions douteuses de salubrité et souvent maltraités. La République refuse ce genre de spectacle où les animaux serviles accomplissent tels des automates, des tours qui ne respectent pas leur nature et n'apprennent rien aux citoyens. La ménagerie républicaine ordonnée par Étienne Geoffroy Saint Hilaire qui y consacre une partie de sa vie, se charge d'offrir un spectacle naturel, pédagogique et édifiant au contraire : un havre pour les compagnons des hommes vaquant le plus librement possible dans un espace commode, tel un livre vivant de l'harmonie retrouvée entre le citoyen régénéré et l'animal protégé ${ }^{61}$.

Las, les policiers n'en ont pas fini avec les espaces clandestins où l'on continue à gagner de l'argent en montrant des animaux. Le 17 frimaire an III, le commissaire est appelé boulevard du Temple sur la plainte du citoyen Lancé qui a été blessé par un taureau. Ce dernier s'est associé à un dénommé Cochon, avec lequel il a loué un local dans lequel se trouve un petit taureau rouge et le ventre blanc, ainsi que trois moutons « intéressants et curieux » et deux singes. Les circonstances de l'accident racontées, il est rappelé l'interdiction de montrer les animaux dans la rue. Finalement la commission administrative de la police de Paris, le 6 nivôse an III, invite le citoyen Cochon à construire une séparation solide entre les spectateurs et l'animal pour prévenir tout incident, avant de délivrer toute autorisation. Le 7 nivôse, la décision se durcit. Finalement il n'aura le droit de montrer que les moutons et les singes, dans une salle sans les promener. Quant au taureau il doit être déposé au Muséum qui le refuse car il est mutilé6 ${ }^{62}$ Par-delà les mesures d'hygiène, de salubrité et de sécurité évidentes, les responsables de l'ordre public soutenus par les constructeurs d'opinion sentent bien que la vision des animaux serviles ne convient pas aux regards des citoyens de la nouvelle république de l'an III. En septembre 1796, le combat du taureau et ses spectacles fut proscrit car on n'y respirait que « lanterne et carnage » selon Pierre de la Mésangère.... En 1800, il est vrai que le régime avait déjà changé, un ancien amphithéâtre, à gauche de l'hôpital Saint Louis, avait déjà ouvert offrant le spectacle de combat d'ours ou de taureau mis à mort par des chiens ${ }^{63}$. Expulser de la rue les animaux exotiques pour les « renaturer » dans la ménagerie du Muséum fait donc partie d'un vaste

(61) Cf Pierre SERNA, « The Republican Menagerie : Animal Politics in the French Revolution » à paraitre dans French History special issue French animal studies, 2014, Pater SAHLINS and Creas PEARSON (dir.).

(62) APP, AA 140, Section fidélité, affaire du 17 frimaire au 8 nivose an III.

(63) Pierre de LA MESANGÈRE, Le voyageur à Paris, tableau pittoresque et moral de cette capitale, Paris, Devaux, an VIII, p. 178. 
plan de régénération des mœurs auquel la police du spectacle de rue doit contribuer au premier chef, en ordonnant le regard possible du citoyen, en enlevant de ses yeux toute forme de violence dégradant les êtres vivants. Protéger les animaux des hommes avides, libérer les humains de leurs plus bas instincts, a constitué une mesure de police supplémentaire peu connue, mais bien réelle au moment où le Directoire tente par bien des moyens, d'inventer le socle d'une civilisation républicaine qui va en partie échouer... à cause des Anglais comme il se doit... et des crypto jacobins évidemment. Une clé de lecture inventée par un lecteur du Journal d'Économie Politique Publique et Morale explique comment le spectacle dégradant des animaux est une peste anglaise relayée par les jacobins ! 1: Les Anglais ont été les premiers à fonder des sociétés politiques pareilles aux jacobins, (les jacobites ou jacobins ne furent ils pas les premiers Tories ?) 2 : Ils ont introduit en France la manie des paris. 3 : Ils sont assez friands de combats atroces pour faire combattre des hommes dans le boxing ou boxe $4:$ Ils énervent ces hommes après avoir agacé les chiens qui se battent. $5:$ Il faut donc interdire toute sorte de spectacle violent au moment où la France sort de « de l'hécatombe jacobine dont les anglais sont les fondateurs!» ${ }^{64}$ cqfd... Confusément ou de façon calculée, le spectacle du sang, du combat, de la discorde est ramené au temps de la guerre civile de 1794. Pour le bon bourgeois Roederer, rédacteur en chef du journal qui laisse publier ce commentaire, un aveu est livré : qu'il le croit ou qu'il joue sur la peur rétrospective, la Terreur et ses exécutions portaient une dimension bestiale de la nature humaine que tout spectacle de violence vient faire remonter à la surface, au risque de l'ordre public. Interdire le combat des animaux violents, c'est refuser l'Ancien Régime et la violence de la guerre civile de 1794. L'animal est bien un acteur de la vie publique et son spectacle, sa fonction, dans l'espace urbain, répondent à une claire volonté politique de l'instrumentaliser pour fonder les valeurs de la République cherchant à inventer une rue pacifiée, une rue propre avec des animaux utiles et instructifs.

L'analyse exhaustive des 633 enquêtes et la lecture de leurs procèsverbaux montrent une quadruple action de l'État en construction, ainsi que la refondation de sa police citoyenne et républicaine, prise dans son travail routinier avec la surveillance normale des accidents qui peuvent

(64) Journal d'économie publique de morale et de politique rédigé par Roederer, tome cinquième, Paris, an V, lettre signée de Monier aîné (je remercie Malik Mellah de m'avoir signalé cet article). 
mener à des débordements, révélateurs des tensions sociales et des enjeux politiques. Le cheval est ce vecteur potentiel de désordres polysémiques.

Le second apport est l'organisation du marché des subsistances, repensée du fait de la refondation de l'économie politique du pays. L'animal mort, à consommer, est un souci récurent du policier et l'économie dirigée de la Terreur l'a démontré. Toutes les contradictions constitutives de l'économie politique de cette période le prouvent : mutation de la boucherie, changement des règles du marché et de la concurrence, réglementation de l'État demandée par les professionnels eux-mêmes, réorganisation complète du métier en fonction des lieux de tueries et de vente des quartiers de viande, représentent autant d'éléments que révèle l'activité abondante de la police en 1793-1794. Le troisième temps est celui de l'élaboration naissante de l'État administrant, l'État enquêtant et l'État inventeur de statistiques liées à l'État protecteur de la santé publique. Seule l'analyse quantifiée des enquêtes pouvait mettre à jour ces trois temps qui disent, au travers de l'étude de la police des animaux, la plus grande histoire de la réorganisation permanente de Paris, l'histoire de l'économie politique, et l'histoire en creux de la naissance de l'État dans sa dimension policière régulatrice. Les animaux, comme les humains, n'échappent pas à la Révolution, ni à la politique. Ils en sont les instruments et les objets, au plus bas de l'échelle des vivants. Au ras du sol, ils offrent un spectacle qui rend compte de la politique au sommet de l'État, selon qu'ils sont montrés comme des créatures en cage, réduits à l'état d'automates animés, ou selon qu'ils sont conservés pour l'éducation du peuple dans un jardin qui conserve au mieux leur liberté naturelle pour les plus inoffensifs. À la condition que la police agisse, à la condition que les citoyens soient convaincus que l'intérêt général de tous les êtres vivants prime sur les intérêts des particuliers. Revient alors en mémoire la scène initiale et le courage de cette femme protégeant le cheval battu, face au silence lâche des hommes, faisant acte de belle et spontanée police. Agissait-elle comme un être éduqué ? Ou comme un être humain instinctif ? On souhaiterait que ce soit comme une femme citoyenne, mais on ne le sait pas. 
ANNEXES

Tableau 3. : Dénominations des animaux

$\begin{array}{ll}\text { ANIMAL } & \text { Effectif } \\ \text { cheval } & 303 \\ \text { viande de boucherie } & 134 \\ \text { chien } & 44 \\ \text { bêtes à cornes } & 34 \\ \text { cochon } & 23 \\ \text { vache } & 20 \\ \text { bœuf } & 17 \\ \text { cochon ladre } & 11 \\ \text { âne } & 5 \\ \text { chèvre } & 4 \\ \text { lapins } & 4 \\ \text { veau } & 3 \\ \text { moutons } & 3 \\ \text { volaille } & 3 \\ \text { viande de veaux } & 2 \\ \text { bœeuf et cheval } & 2 \\ \text { agneaux et cochons } & 2 \\ \text { animaux de foire } & 2 \\ \text { perroquet } & 2 \\ \text { X } & 2 \\ \text { poules lapins cochons } & 2 \\ \text { harengs } & 2 \\ \text { poule } & 2 \\ \text { viande de boucherie/suif } & 1 \\ \text { viande de boucherie/mouton } & 1 \\ \text { tripes } & 1 \\ \text { bestiaux } & 1 \\ \text { taureau } & 1 \\ \text { chiens } & 1 \\ \text { cochon chats chien } & \text { éléphant } \\ \text { bêtes féroces } & 1 \\ \text { bêtes fauves } & 1 \\ \text { os et poisson gâté } & 1 \\ \text { matières animales } & 1 \\ \text { suifs } & 1 \\ \text { pigeons } & 1 \\ \text { coq } & 1 \\ \text { volaille et gibier } & 1 \\ \end{array}$


Pierre SERNA Directeur de l'IHRF Paris I Panthéon Sorbonne 79 rue du faubourg Saint-Vincent 45000 Orléans pierreserna@wanadoo.fr avec Julien ALÉRINI pour les graphiques 